\title{
Breve introducción a la obra de A. N. Kolmogorov (1903-1987)
}

\section{Francisco Javier Girón}

Arbor CLXXVIII, 704 (Agosto 2004), 583-588 pp.

\section{Introducción}

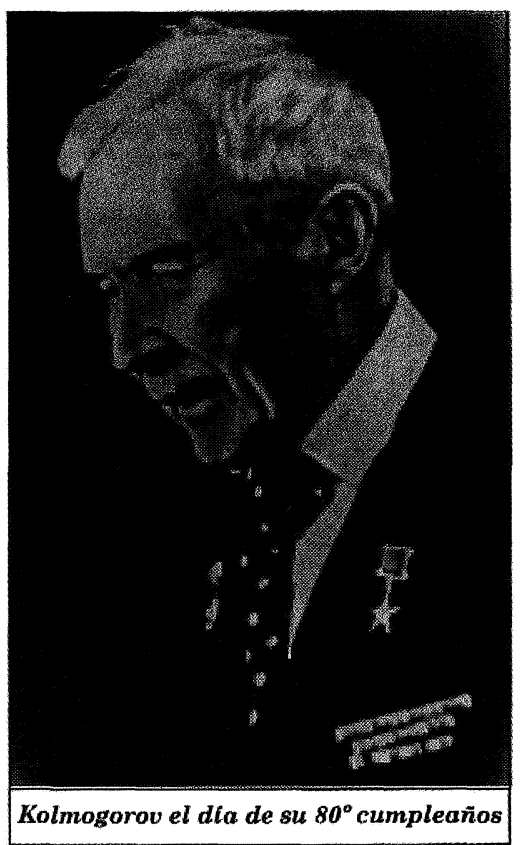

Dentro de las actividades científicas de la Sección de Exactas de la Real Academia de Ciencias a lo largo del curso 2003-2004, el día 2 de febrero de 2004 se conmemoró el centenario del nacimiento de Andrei Nikolayevich Kolmogorov, uno de los más grandes matemáticos del siglo $\mathrm{XX}$, con un breve ciclo de dos conferencias a cargo de dos especialistas en sus respectivas materias, precedidas de una breve introducción biográfica del autor de estas líneas y coordinador del ciclo, prof. Javier Girón. La primera de las conferencias corrió a cargo del profesor Javier Jiménez Sendín de la Universidad Politécnica de Madrid sobre la teoría de la turbulencia, una de las grandes aportaciones de Kolmogorov, y la segunda

a cargo del prof. David Nualart de la Universidad Central de Barcelona 
sobre el cálculo de probabilidades y la teoría de los procesos estocásticos quizás la contribución más importante de Kolmogorov a las matemáticas ya que sentó las bases axiomáticas de estas dos disciplinas. La Sección de Exactas desea agradecer a estos dos renombrados especialistas su participación el el ciclo.

\section{Breve reseña científico-biográfica}

Kolmogorov nació el 25 de Abril de 1903. En 1920 fue a estudiar matemáticas a Moscú bajo la supervisión de Stepanov. En 1922 consiguió su primer resultado importante que demuestra que no existe una tasa de convergencia a cero, lo más lenta posible, de los coeficientes de Fourier de una función integrable. En ese mismo año, con solo 19 años, construyó el primer ejemplo de una función integrable cuya serie de Fourier diverge casi por doquier y refinó el resultado desde el casi por doquier a todo punto, lo que le hizo universalmente famoso.

En 1925 escribe un artículo con Jinchin en el que demuestran por vez primera el teorema de las tres series.

Poco más tarde, como alumno de doctorado de Luzin, publicó 18 artículos sobre cálculo de probabilidades y lógica intuicionista. En 1931 sentó las bases de la teoría moderna de los procesos de difusión y los relacionó con la teoría de las ecuaciones en derivadas parciales. En ese mismo año fue nombrado catedrático de la Universidad de Moscú.

Publica su famosa monografía sobre los fundamentos del cálculo de probabilidades en alemán Grundbegriffe der Wahrscheinlichkeitsrechnnung en 1933, escrita en un bosque junto a la ribera de un riachuelo. El artículo de David Nualart en este volumen examinará con detalle la importancia de esta obra y de otras cotribuciones de Kolmogorov a la Teoría de la Probabilidad y a los Procesos Estocásticos.

En la década de los años 30 del siglo $\mathrm{XX}$, además de proseguir con su trabajo en la teoría de la probabilidad, desarrolló la teoría de la cohomología, la teoría de la reversibildad estadística y, en colaboración con Gelfand, la teoría de los anillos de funciones continuas sobre espacios topológicos, entre otras numerosas aportaciones.

De 1938 procede uno de los trabajos más influyentes de Kolmogorov sobre el suavizado y la predicción de procesos estocásticos. En 1939 la Academia de Ciencias de la Unión Soviética lo elige como académico y poco más tarde será nombrado secretario del departamento de ciencias físicas 
y matemáticas de la Academia. De 1940 es su famoso artículo sobre la turbulencia, tema en el que siguió trabajando durante un largo período y que se comenta con detalle en el artículo de Javier Jiménez Sendín en este número monográfico.

Desde el punto de vista personal queremos señalar que, en 1942, Kolmogorov se casa con Anna Dimitrievna Egovora.

En 1946 es nombrado director del Laboratorio de Turbulencia del Instituto Académico de Geofísica Teórica y, en el bienio 1970-72, a la manera del capitán Cook, zarpó en una vuelta al mundo en el barco oceanográfico Dimitri Mendeleyev, como supervisor científico de un estudio sobre la turbulencia oceánica.

En los años de la posguerra publicó artículos sobre geología matemática, inferencia estadística y procesos de ramificación y publicó un libro con Gnedenko, que pronto se convertiría en un clásico, titulado The Limit Distributons for Sums of Independent Random Variables. En 1951 publicó un artículo sobre cadenas de Markov en tiempo continuo que generó una cantidad inmensa de literatura sobre el tema.

Prácticamente, Kolmogorov no dejó sin explorar ninguno de los campos de la Matemática del siglo XX, y en muchos de ellos realizó aportaciones fundamentales. Una lista, amplia pero no exhaustiva de las áreas de la Mátemática donde dejó su impronta, incluiría las siguientes: la teoría de las series trigonométricas, la teoría de la medida, la teoría de los conjuntos, la teoría de la integración, la lógica constructiva (el intuicionismo), la topología, la teoría de la aproximación, la teoría de la probabilidad y la teoría de los procesos estocásticos que se examinan con detalle en el artículo de D. Nualart, la teoría de la información, la estadística matemática a la que dedicaremos una breve sección dentro de esta nota introductoria, los sistemas dinámicos, la teoría de autómatas, la teoría de algoritmos, la lingüística matemática, la teoría de la turbulencia que será desarrollada en el artículo de J. Jiménez Sendín, la mecánica celeste, la teoría de las ecuaciones diferenciales, su aportación al decimotercer problema de Hilbert y a la balística. No debemos de olvidar las aplicaciones de las matemáticas a problemas de la Biología, la Geología, y a la cristalización de metales, en las que siempre estuvo interesado.

Desde 1950 en adelante los trabajos científicos más importantes de Kolmogorov se centraron alrededor de cuatro ideas fundamentales: probabilidad, dinámica, información y complejidad. Su otra actividad importante fue la educación matemática a la que dedicó una gran atención hasta sus últimos días.

Kolmogorov fue una persona que mostró un gran respeto y admiración 
por otros grandes matemáticos de su época como Fréchet, a quién llamaba su maestro, Doob y Fisher.

Falleció el 20 de Octubre de 1987.

\section{Contribuciones a la Estadística Matemática}

Aunque la importancia de las aportaciones de Kolmogorov a la Estadísitica Matemática no han tenido la misma repercusión que sus grandes contribuciones a la Teoría de la probabilidad o al estudio de la Turbulencia, algunos de sus trabajos sobre la estadística matemática han tenido una gran influencia en el desarrollo de esta disciplina y en algunas áreas de la ingeniería y de la econometría.

La aportación de Kolmogorov a la estadística no paramétrica se refleja en los llamados tests o contrastes de Kolmogorov (1933) y de KolmogorovSmirnov (1939) para los llamados problemas de una y dos muestras independientes, respectivamente, que suponen una importante extensión del clásico teorema de Glivenko-Cantelli y permiten cuantificar la discrepancia entre la distribución empírica y la hipótesis nula, en un caso y la discrepancia entre dos distribuciones empíricas, respectivamente.

El resultado se establece para el primero de los problemas de la siguiente manera:

Si $D_{n}(x)=\sup \left|F_{n}(x)-F(x)\right|$, donde $F_{n}(x)$ es la distribución empírica y $F(x)$ es continua, entonces

$$
\lim _{n \rightarrow \infty} \operatorname{Pr}\left(\sqrt{n} D_{n}<\lambda\right)=Q(\lambda)=\sum_{k=-\infty}^{\infty}(-1)^{k} \exp \left(-2 k^{2} \lambda^{2}\right) \quad \text { si } \quad \lambda>0 .
$$

A la distribución límite $Q(\lambda)$, que no depende de $F$, se la conoce con el nombre de distribución de Kolmogorov-Smirnov (1939).

En 1942, Kolmogorov introdujo el concepto de suficiencia parcial, o $\mathrm{K}$-suficiencia, relacionado con la suficiencia parcial en presencia de parámetros marginales de la suficiencia bayesiana.

Uno de los trabajos más importantes de Kolmogorov de finales de la década de los años treinta del siglo XX, concretamente del año 1938, fue 
el relacionado con el suavizado y predicción de series temporales estacionarias.

Estos trabajos están muy relacionados con los que llevó a cabo en Estados Unidos Norbert Wiener sobre el mismo tema y aproximadamente en la misma época; estos se desarrollaron de modo independiente pero complementario, y se mantuvieron en secreto debido a su importancia militar, aunque al parecer no hay duda de que Kolmogorov fue el primero en publicar su trabajo.

Este trabajo supuso una gran revolución y tuvo una influencia inmensa al establecer un nexo entre los probabilistas y los estadísticos, e inició una nueva rama de la ingeniería y de la economía.

El paso del estudio de los procesos estacionarios a los campos estocásti$\cos$, y de estos al estudio de la turbulencia era el paso natural que condujo a Kolmogorov a uno de sus descubrimientos más importantes en 1940.

Señalamos, por último, un artículo de Kolmogorov sobre la teoría de los estimadores insesgados de 1950, que demuestra su conocimiento de los resultados teóricos que se estaban produciendo por esas fechas en la teoría de los estimadores insesgados.

En el artículo, utiliza el famoso teorema de Rao-Blackwell (1945-47) para calcular un estimador insesgado de la proporción

$$
\pi=\Phi\left(\frac{c-\mu}{\sigma}\right)
$$

de los valores que están por debajo de un valor $c$ en una población normal de parámetros $\mu$ y $\sigma$ desconocidos, problema de gran importancia práctica.

\section{Kolmogorov y la educación matemática}

Durante la etapa en la que Kolmogorov se dedicó a la complejidad, a partir de la década de los 50, se interesó de forma muy activa en los problemas de la educación matemática, sobre todo en la creación de escuelas para niños superdotados, y en especial en la patrocinada por la Universidad del Estado de Moscú.

El poner en marcha este programa se debió al gran prestigio de que gozó Kolmogorov entre la clase política, a pesar de no haber participado nunca en élla. 
En esa escuela, en la que Kolmogorov impartió 24 horas semanales de clase durante muchos años - además de elaborar planes de estudio y escribir libros-, enseñaba literatura, poesía, arte y música, y organizaba excursiones y marchas. Su idea era ampliar y abrir nuevos horizontes que moldearan la personalidad de los niños, sin importarle que más adelante fuesen matemáticos. Lo que realmente le importaba era que en su futura profesión, cualquiera que fuese, mantuvieran una gran amplitud de miras y tuvieran una curiosidad ilimitada. bor.

Así dejó sentados los cimientos para que otros continuasen con su la-

\section{Epílogo}

Alexandrov, con motivo del 50 cumpleaños de Kolmogorov, comenta lo siguiente:

Kolmogorov pertenece a ese reducido grupo de matemáticos para los que cualquier publicación en cualquier área conduce a una revisión completa de la misma. Es difícil en esta época (1953) encontrar a un matemático que se interese en tantos campos de especialización, y que haya tenido tanta influencia en el desarrollo de las matemáticas ... baste decir que Hardy lo tomó por un especialista en series trigonométricas y von Harman por un especialista en mecánica. 